\title{
Four-Dimensional Thoracic CT in Free-Breathing Children
}

\author{
Hyun Woo Goo, MD, PhD \\ Department of Radiology and Research Institute of Radiology, Asan Medical Center, University of Ulsan College of Medicine, Seoul, Korea
}

In pediatric thoracic CT, respiratory motion is generally treated as a motion artifact degrading the image quality. Conversely, respiratory motion in the thorax can be used to answer important clinical questions, that cannot be assessed adequately via conventional static thoracic CT, by utilizing four-dimensional (4D) CT. However, clinical experiences of 4D thoracic CT are quite limited. In order to use 4D thoracic CT properly, imagers should understand imaging techniques, radiation dose optimization methods, and normal as well as typical abnormal imaging appearances. In this article, the imaging techniques of pediatric thoracic 4D CT are reviewed with an emphasis on radiation dose. In addition, several clinical applications of pediatric $4 \mathrm{D}$ thoracic $\mathrm{CT}$ are addressed in various thoracic functional abnormalities, including upper airway obstruction, tracheobronchomalacia, pulmonary air trapping, abnormal diaphragmatic motion, and tumor invasion. One may further explore the clinical usefulness of $4 D$ thoracic $C T$ in free-breathing children, which can enrich one's clinical practice.

Keywords: 4D CT; CT imaging techniques; Chest CT; Child; Airway; Tracheobronchomalacia; Air trapping;

Lung densitometry; Diaphragm motion; Tumor invasion

\section{INTRODUCTION}

Respiratory motion artifacts often degrade image quality of three-dimensional (3D) static thoracic CT in freebreathing children. To decrease the artifacts, general anesthesia with intubation (1), controlled ventilation (2), or respiratory triggering $(3,4)$ may be used. Conversely, respiratory motion during multi-phase respiratory dynamic, the so-called four-dimensional (4D) CT scanning actually can be used to answer clinical questions. 4D CT imaging technique is widely used in perfusion CT imaging (5) and radiation oncology (6). As a preclinical study, 4D CT dataset has been used to generate lung ventilation imaging (7). However, its applications in thoracic imaging are quite limited and generally include the evaluation of airways and diaphragms (8-16). The high radiation dose

Received May 19, 2018; accepted after revision July 31, 2018. Corresponding author: Hyun Woo Goo, MD, PhD, Department of Radiology and Research Institute of Radiology, Asan Medical Center, University of Ulsan College of Medicine, 88 Olympic-ro 43gil, Songpa-gu, Seoul 05505, Korea.

- Tel: (822) 3010-4388 - Fax: (822) 476-0090

- E-mail: ghw68@hanmail.net

This is an Open Access article distributed under the terms of the Creative Commons Attribution Non-Commercial License (https://creativecommons.org/licenses/by-nc/4.0) which permits unrestricted non-commercial use, distribution, and reproduction in any medium, provided the original work is properly cited. from retrospectively respiratory-gated $4 \mathrm{D}$ thoracic $\mathrm{CT}$ (8) deems the imaging technique inappropriate in diagnostic pediatric imaging. Hence, 4D CT without respiratory gating should be used in children (9-16). Moreover, the radiation dose of clinically indicated pediatric $4 \mathrm{D}$ thoracic CT should be optimized by utilizing various radiation dose reduction techniques (17). In this review, optimal pediatric 4D thoracic CT imaging techniques are first described and several clinical applications, including upper airway obstruction, tracheobronchomalacia, pulmonary air trapping, and diaphragmatic motion abnormalities, are subsequently illustrated with typical clinical examples.

\section{Imaging Techniques}

Four-dimensional thoracic CT scanning is defined as multiple consecutive 3D thoracic $\mathrm{CT}$ data acquisitions with the same longitudinal scan range, and without CT table movement throughout the respiratory cycle. Although all or some of the pediatric patients were intubated and their respiratory rates were artificially manipulated $(40 / \mathrm{min})$ in two 4D pediatric thoracic CT studies $(9,14), 4 \mathrm{D}$ CT was generally acquired during free breathing $(10-13,16)$. Scan time of $4 \mathrm{D}$ thoracic $\mathrm{CT}$ should be adjusted to include at least one respiratory cycle. In pediatric patients, the scan time was varied in the range of 2.0-4.6 seconds, depending 
on patients' respiratory rates $(9-14,16)$. The longitudinal coverage of $4 \mathrm{DCT}$ is largely in the range of $3.8 \mathrm{~cm}(64 \times 0.6$ $\mathrm{mm})-16 \mathrm{~cm}(320 \times 0.5 \mathrm{~mm}$ or $256 \times 0.625 \mathrm{~mm})$ depending on detector configurations of the CT scanners. In our institution, 4D CT is performed with a second-generation dual-source CT scanner (SOMATOM Definition Flash; Siemens Healthineers, Forchheim, Germany). In other institutions, a 320-detector row CT scanner (Aquilion ONE; Canon Medical Systems, Otawara, Japan) was frequently used for pediatric $4 \mathrm{D}$ thoracic $\mathrm{CT}(9,13,14)$. Considering the differences in hardware and software, imaging parameters of pediatric $4 \mathrm{D}$ thoracic CT should be individually optimized for a specific CT scanner.

Two types of scan modes, i.e., intermittent and continuous modes, are available for 4D CT scanning in the scanner (SOMATOM Definition Flash) (Fig. 1). The differences between the two scan modes are the phase numbers (six vs. nine in 2 seconds) and the presence or absence of inter-phase time intervals (approximately 123msec interval vs. no interval). Other imaging parameters are the same as follows: $64 \times 0.6 \mathrm{~mm}$ slices, a $0.75-\mathrm{mm}$ slice width, a $0.4-\mathrm{mm}$ reconstruction interval, a z-axis coverage of $3.8 \mathrm{~cm}$, a temporal resolution of $210 \mathrm{msec}$, and total scan time of 2 seconds. Because the dose-length product value of the continuous mode is four-times higher than that of the intermittent mode at the same tube voltage and current setting (Fig. 1), the intermittent mode is used for pediatric thoracic $4 \mathrm{DCT}$ to reduce radiation dose. The radiation dose difference between the two scan modes is attributable to the $180^{\circ}$ partial scan in the intermittent mode and the $360^{\circ}$ full scan in the continuous mode, in addition to the difference in the number of phases. Six phases in 2 seconds (three phases per second) in the intermittent mode usually include more than one respiratory cycle in young children, and hence the qualitative evaluation of pediatric 4D thoracic CT is feasible (16). Such information regarding the number of phases per second is lacking in other pediatric $4 \mathrm{D}$ thoracic CT studies. On the other hand, $4 \mathrm{D}$ thoracic $\mathrm{CT}$ using a 320-slice CT scanner was performed with image acquisition of six phases per second in approximately 7 seconds to cover two respiratory cycles in eight adult patients with lung cancer (18). Such an adult protocol delivering high radiation dose should be modified to a pediatric protocol with particular attention to radiation dose.

Low radiation dose settings with diagnostic image quality is generally possible in pediatric $4 \mathrm{D}$ thoracic $\mathrm{CT}$, because of the inherently high air-tissue contrast in the thorax. To maintain sufficient image quality among patients with different body habitus, a body size-adaptive protocol should be used in pediatric $4 \mathrm{D}$ thoracic $\mathrm{CT}$, as in the other pediatric CT imaging techniques. In our institution, radiation dose of pediatric thoracic $\mathrm{CT}$ is individually determined, based on the cross-sectional area and mean body density measured on an axial CT image obtained approximately $1-2 \mathrm{~cm}$ above the dome of the liver (19). Other body size indices, such as body weight and body mass index, are used to adjust radiation dose of pediatric $4 \mathrm{D}$ thoracic $\mathrm{CT}$ in others. To avoid high image noise at 70 or $80 \mathrm{kV}, 100 \mathrm{kV}$ is used for pediatric $4 \mathrm{D}$ thoracic $\mathrm{CT}$ in our institution, while others utilize $80 \mathrm{kV}$ partly for the combined evaluation of vascular airway compression, after the intravenous administration of iodinated contrast agent $(9,13,14)$. It is noteworthy that the lower limit of radiation dose at $100 \mathrm{kV}$ is present in the second-generation dual-source $\mathrm{CT}$, and radiation dose,

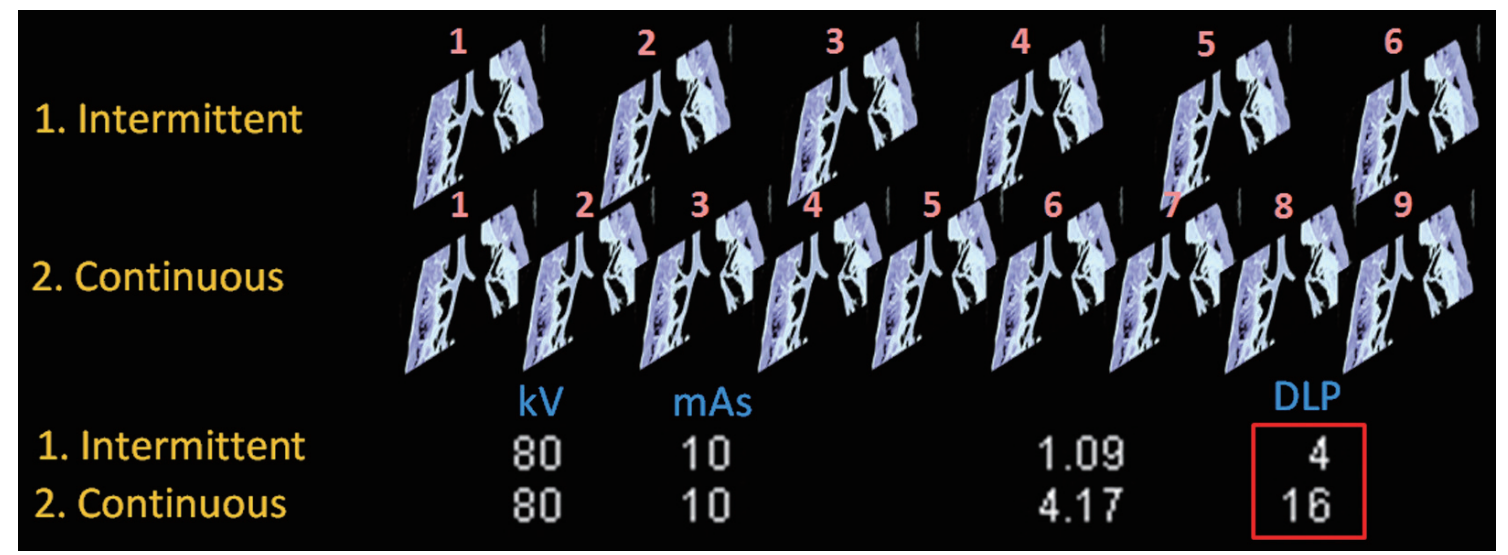

Fig. 1. Comparison between intermittent and continuous scan modes of 4D pediatric thoracic CT. Six intermittent and nine continuous sequential scans are performed in 2 seconds. At same $80 \mathrm{kV}$ and $10 \mathrm{mAs}$, DLP value of continuous scan mode is $16 \mathrm{mGy} \cdot \mathrm{cm}$, which is four-times higher than that of intermittent mode $(4 \mathrm{mGy} \cdot \mathrm{cm}) . \mathrm{DLP}=$ dose length product, $4 \mathrm{D}=$ four-dimensional 
therefore, cannot be reduced below $1.2 \mathrm{mGy}$ in volume CT dose index, in majority of the young children (16). The reported effective dose values of pediatric $4 \mathrm{D}$ thoracic $\mathrm{CT}$ are in the range of $0.4-3.0 \mathrm{mSv}(9,13,14)$. Because of a limited longitudinal coverage (approximately $3.8 \mathrm{~cm}$ ), the scan position of $4 \mathrm{D}$ thoracic $\mathrm{CT}$ should be carefully adjusted on the scout image to include target structures. On the contrary, the scan range needs to be shortened for young children in order to avoid unnecessary radiation exposure, if the scanners with $16-\mathrm{cm}$ longitudinal coverage are utilized.

In the second-generation dual-source scanner, an iterative reconstruction algorithm is not available for $4 \mathrm{D}$ CT. However, if available, an iterative reconstruction algorithm should be used for 4D CT. The scanned thin axial 4D datasets (95 images/phase $\times 6$ phases $=570$ images) are transferred to a workstation (Leonardo; Siemens Healthineers) for dynamic multiplanar and 4D evaluations utilizing a dedicated 4D post-processing software (Inspace; Siemens Healthineers).

\section{Clinical Applications}

\section{Upper Airway Obstruction}

Normal and abnormal dynamic appearances of the pediatric upper airway can be evaluated with $4 \mathrm{D} C \mathrm{CT}$, which is one of the most common clinical applications in newborns and infants (9-14). Generally, the uvula, tongue, epiglottis, vocal cord, and pyriform sinuses serve as anatomic landmarks for image evaluation. Upper airway dimension is variably distended during inspiration and variably collapsed during expiration, depending on the amount of airflow and the strength of the supporting soft tissue structures. Intermittent velopharyngeal closure separating the oral cavity from the nasal cavity may be observed on $4 D$ CT as a normal dynamic finding (Fig. 2). Therefore, 4D CT has the potential to assess velopharyngeal insufficiency caused by various disorders such as cleft palate and neuromuscular disorders. In upper airway obstruction, 4D CT is particularly useful to simply distinguish a dynamic obstructive lesion (Fig. 3) from a fixed one (Fig. 4), which is crucial for treatment planning. Glossoptosis, defined as abnormal posterior displacement of the tongue and commonly seen in children with micrognathia (particularly in Pierre Robin syndrome), Down syndrome, and neurologic impairment, may cause oropharyngeal obstruction and obstructive sleep apnea. The diagnosis of glossoptosis (Fig. 5) can be made with neck lateral view, fluoroscopy, 4D CT, and endoscopy. Radiologists are usually not familiar with normal (Fig. 6) and abnormal laryngeal motion, and hence, a learning curve is necessary for $4 \mathrm{D} \mathrm{CT}$ evaluation in patients with suspected laryngomalacia. Laryngomalacia, the most common cause of inspiratory stridor in the first year of life, is caused by excessive inward collapse of the supraglottic structures. The diagnosis and severity of laryngomalacia can be assessed with fiberoptic nasolaryngoscopy and 4D CT (Fig. 7). It is usually self-limited, but surgical treatment is necessary in a small subset of patients with severe laryngomalacia. 4D CT may also be preferably used to evaluate treatment response in laryngomalacia because of its noninvasive nature (Fig. 7).

\section{Tracheobronchomalacia}

Tracheobronchomalacia is defined as an excessive expiratory collapse of the tracheobronchial tree. In addition to the primary type, the secondary type of tracheobronchomalacia may develop as a result of
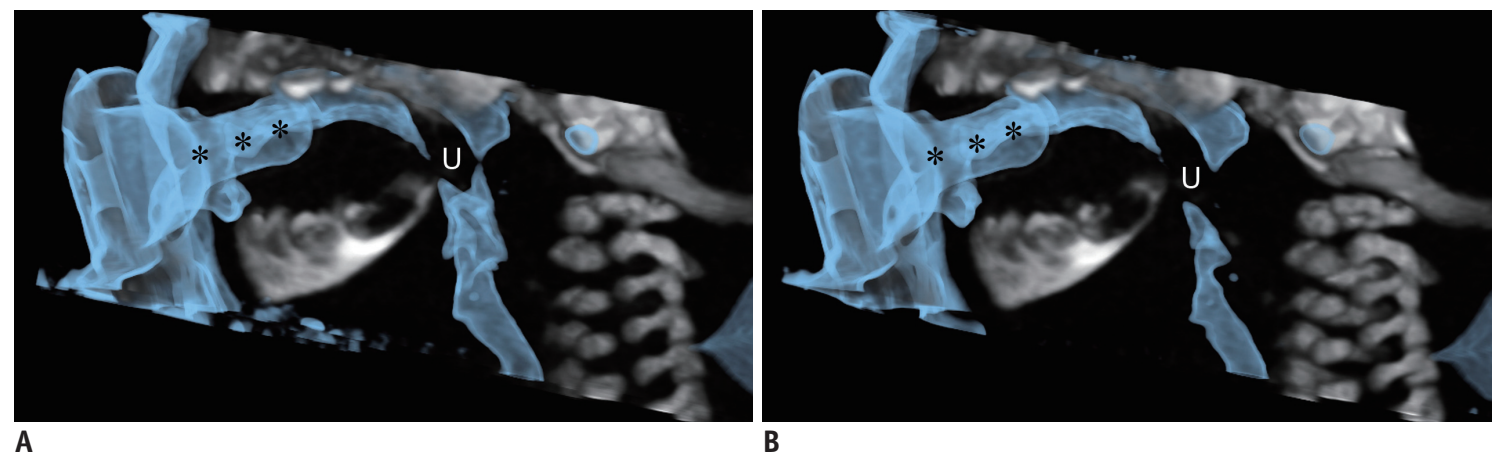

Fig. 2. Normal upper airway in 1-month-old boy with vascular ring.

Sagittal inspiratory (A) and expiratory (B) volume-rendered CT images demonstrate normal appearance of upper airway. U separating nasopharynx from oropharynx is noted. $U$ contributes to velopharyngeal closure with other velopharyngeal muscles that is indispensable for normal swallowing and speaking. As shown in these merged volume-rendered images, bone structures can be added to airway structures to facilitate anatomical correlations. To note, upper airway dimension diminishes normally from inspiration to expiration. Patient was sucking on pacifier (asterisks) during $\mathrm{CT}$ examination. $\mathrm{U}=$ uvula 
several pathophysiologic mechanisms such as tissue injury, inflammation, and longstanding extrinsic compression. Tracheomalacia is commonly associated with tracheoesophageal fistula and therefore should be strongly
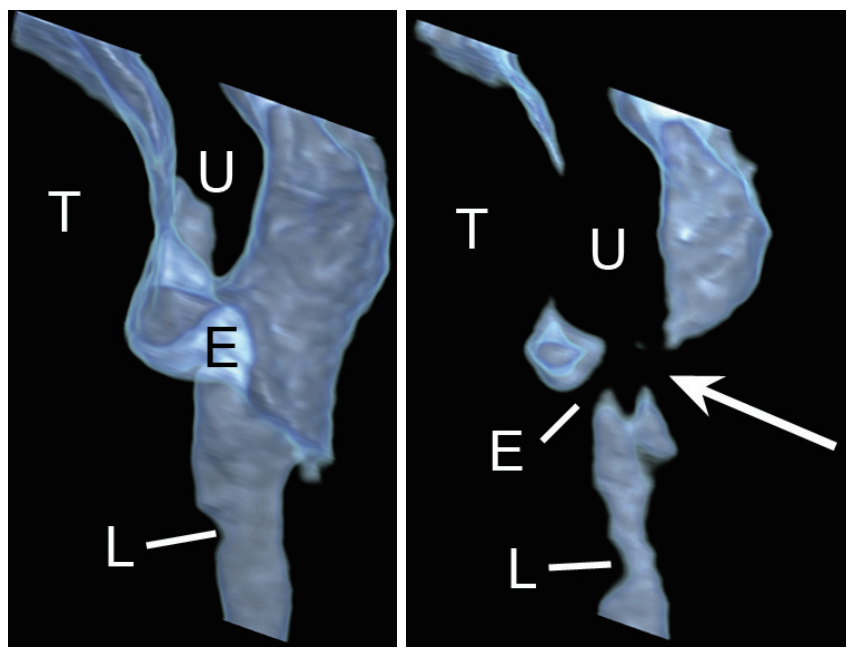

A

B

Fig. 3. Dynamic pharyngeal obstruction in 2-month-old girl with complete atrioventricular septal defect.

Sagittal inspiratory (A) and expiratory (B) volume-rendered CT images reveal severe dynamic expiratory narrowing (arrow) of pharynx.

$\mathrm{E}=$ epiglottis, $\mathrm{L}=\operatorname{larynx}, \mathrm{T}=$ tongue suspected and evaluated for, in patients suffering from postsurgical respiratory symptoms. Paired end-inspiratory/ end-expiratory $\mathrm{CT}$ (1), dynamic expiratory $\mathrm{CT}$, cine $\mathrm{CT}$ (20), or the more recent 4D CT (9-14) (Fig. 8) may be used for the diagnosis of tracheomalacia. Compared to other imaging methods, the evaluation with 4D CT can be performed during free breathing, regarded as a more physiologic state, without additional respiratory maneuvers or procedures. Moreover, its gapless 4D imaging capability can overcome the limitations of cine $\mathrm{CT}$, including difficulties in evaluating longitudinally moving and obliquely coursing structures. As a result, 4D CT allows the accurate evaluation of the extent and severity of tracheomalacia, and it makes the diagnosis of bronchomalacia feasible. Traditionally, an empirical cut-off value of $50 \%$ in a reduction of cross-sectional area has been used for the diagnosis of tracheomalacia, not only in adults, but also in children (1). However, its diagnostic value is compromised by the fact that the tracheal collapsibility in normal subjects may also be greater than $50 \%$. In a comparative study between 27 children with bronchoscopy-proven tracheomalacia and 320 control patients (20), a diagnostic cut-off value of $31.6 \%$ in tracheal collapsibility on free-breathing cine $\mathrm{CT}$ was recently

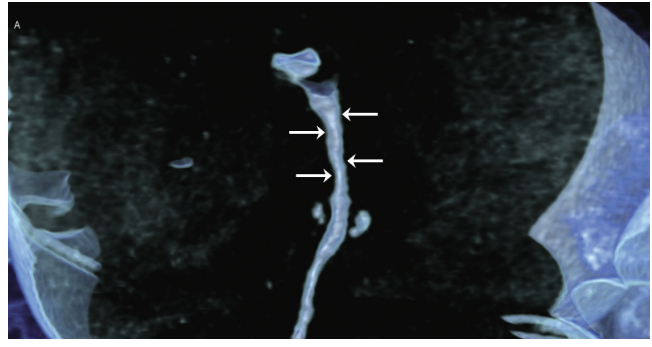

A
B

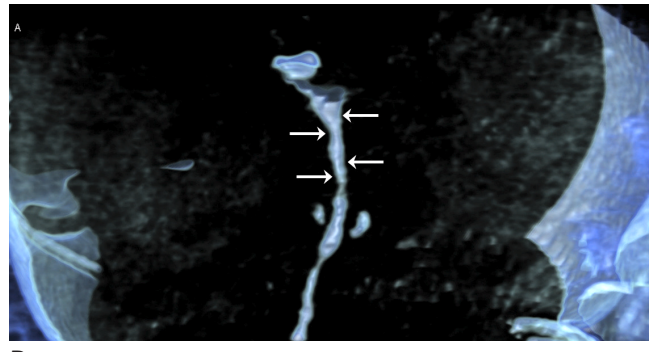

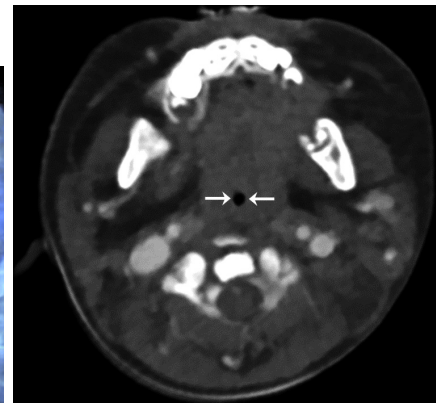

C

Fig. 4. Fixed obstruction of entire pharynx in 9-month-old boy with repaired hypoplastic left heart syndrome.

Oblique coronal inspiratory (A) and expiratory (B) volume-rendered CT images show diffuse, fixed pharyngeal narrowing (arrows). C. Axial contrast-enhanced CT image reveals soft tissue thickening causing pharyngeal narrowing (arrows).
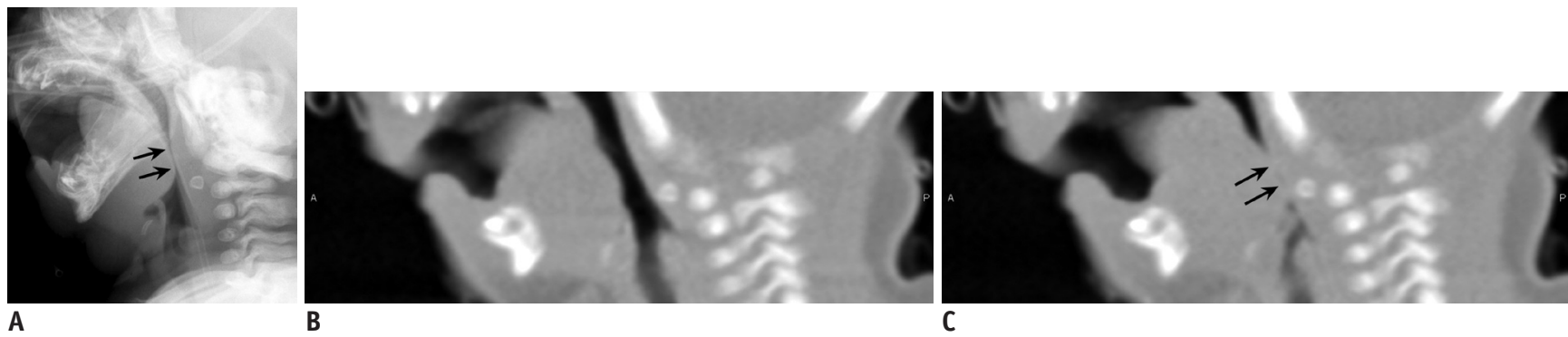

C

Fig. 5. Glossoptosis in 1-month-old boy with repaired diaphragmatic hernia and multiple congenital anomalies.

A. Neck lateral radiograph shows abnormal posterior displacement of tongue causing pharyngeal obstruction (arrows). Sagittal thick-slab inspiratory (B) and expiratory (C) CT images demonstrate that pharynx is patent during inspiration but completely obliterated (arrows) during expiration. $\mathrm{A}=$ anterior, $\mathrm{P}=$ posterior 
suggested with $96.3 \%$ sensitivity, $97.2 \%$ specificity, and $97.1 \%$ accuracy for the diagnosis of tracheomalacia. In line with the result, tracheal collapsibility on free-breathing $4 \mathrm{D}$ CT was less than $34 \%$ in normal pediatric patients and in
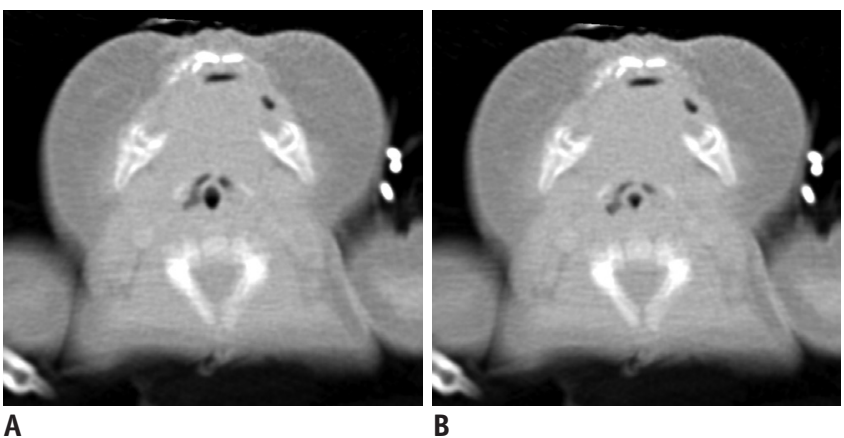

Fig. 6. Normal larynx in 3-month-old boy with tetralogy of Fallot and suspected laryngomalacia.

Axial inspiratory (A) and expiratory (B) thick-slab CT images show normal laryngeal appearance during respiration. the range of $43-90 \%$ in patients with malacic airways (13). One of the current problems is that time-consuming manual airway tracing on hundreds to thousands of $4 \mathrm{D}$ CT images is necessary to evaluate airway collapsibility. Therefore, to enhance the clinical utility of $4 D C T$ for the diagnosis of tracheobronchomalacia, it is imperative that an automatic or semiautomatic quantitative evaluation algorithm needs to be developed.

\section{Pulmonary Air Trapping}

Similar to free-breathing cine CT (21), densitometric analysis of the lung parenchyma is feasible with freebreathing 4D CT. Consequently, we can easily differentiate a lung lesion with air trapping, from a normally ventilated lung region on $4 \mathrm{D}$ volume-rendered $\mathrm{CT}$ images (Fig. 9). It may be interesting to note that we may accentuate the visualization of lung ventilation, which is normally greater

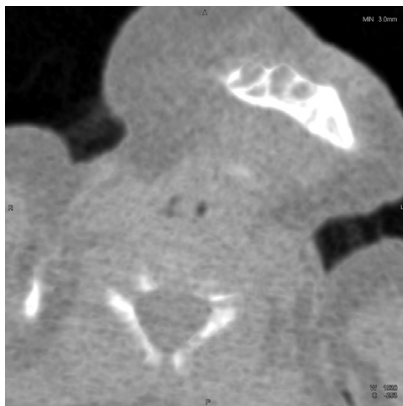

A

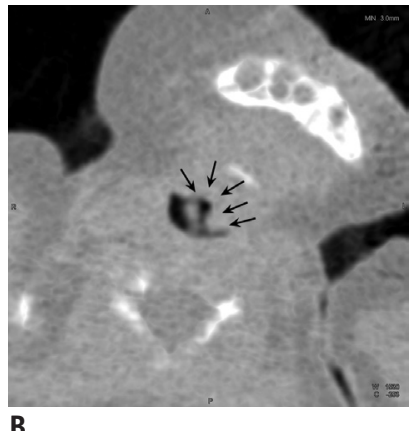

B

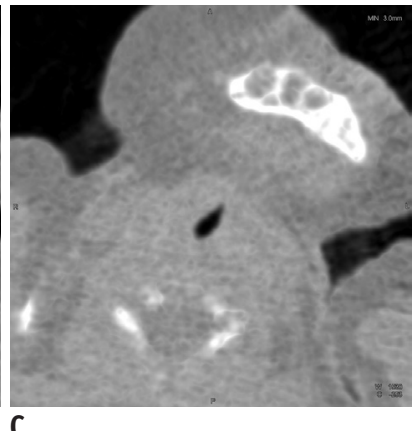

C
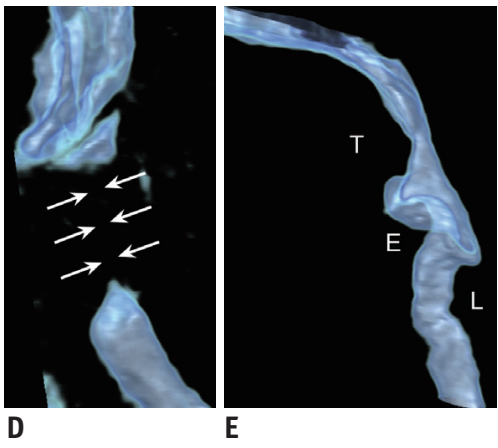

Fig. 7. Laryngomalacia in 1-month-old girl with tetralogy of Fallot and inspiratory stridor.

A. Axial inspiratory thick-slab minimum intensity projection CT image shows collapsed larynx due to severe laryngomalacia. B. Axial thickslab minimum intensity projection CT image obtained between inspiration and expiration illustrates typical omega-shaped epiglottis (arrows) caused by inward collapse of aryepiglottic folds. C. Axial expiratory thick-slab minimum intensity projection CT image displays intact laryngeal shape. D. Sagittal inspiratory volume-rendered CT image illustrates excessive inspiratory collapse of supraglottic airway (arrows) due to severe laryngomalacia. E. Sagittal inspiratory volume-rendered CT image shows improvement of laryngomalacia after epiglottopexy.

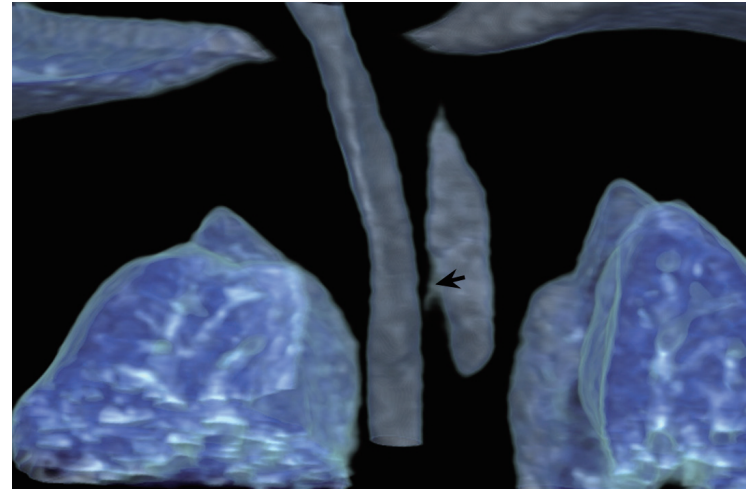

A

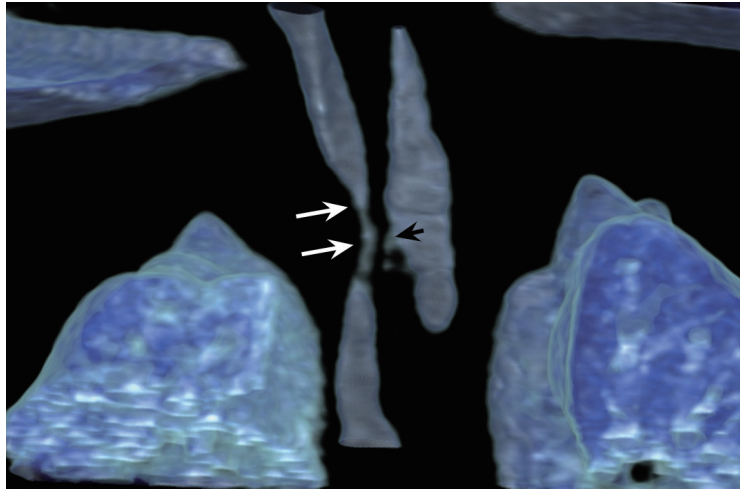

B

Fig. 8. Tracheomalacia in 2-month-old boy with tetralogy of Fallot after esophagoesophagostomy for esophageal atresia and ligated tracheoesophageal fistula.

Coronal inspiratory (A) and expiratory (B) volume-rendered CT images show severe expiratory tracheal collapse (long arrows) indicating tracheomalacia at thoracic inlet level. Longitudinal extent of focal tracheomalacia is nicely illustrated on expiratory-phase 4D CT image (B). Remnant of ligated tracheoesophageal fistula (short arrows) is noted. 
in the posterior dependent lungs, by freely adjusting the opacity of 4D CT images. Nevertheless, 4D CT-based lung ventilation imaging using a deformable image registration algorithm is desirable for the accurate and reproducible quantitative evaluation of regional ventilation function (22).

\section{Diaphragm Motion}

For diaphragm motion analysis, fluoroscopy, sonography, and magnetic resonance imaging (MRI) have all been used $(11,23)$. M-mode and B-mode sonography is most commonly used for diaphragmatic motion analysis in children, but the imaging method is limited by operator dependency and obscured acoustic window (24). A recent study (16) demonstrates that the diaphragmatic motion analysis and the densitometric analysis of adjacent lung parenchyma are feasible with free-breathing $4 \mathrm{D} \mathrm{CT}$, and the diagnosis of diaphragm paralysis or palsy is attainable in select children. In the literature, the normal diaphragm motion on M-mode sonography is defined as the respiratory excursion greater than $4 \mathrm{~mm}$ and the difference of the excursions between both sides less than $50 \%$, irrespective of patient size and the degree of lung inflation (24). The lack of body size- and respiration-adapted normative data substantially hampers the accurate analysis of diaphragm motion in children. Hence, it is imperative to establish such normal data. In addition, a mobile CT unit capable of 4D CT scanning should be available to be used routinely in intensive care units.

\section{Tumor Invasion}

To evaluate chest wall and aortic invasion by thoracic neoplasms pre-operatively, respiratory dynamic MRI has been used $(25,26)$. Dynamic 4D CT may also be used for this purpose. In the initial clinical experience in a small number of adult patients with lung cancer (18), 4D CT could reveal differential movements between the tumor and the adjacent structures in seven patients without direct invasion, as well as a small area of fixation due to superficial invasion of the adventitia of the superior vena cava in one case. There has been no such 4D CT study in children. Further clinical experiences are warranted to substantiate the clinical value of $4 \mathrm{D}$ CT in identifying preoperative thoracic tumor invasion.

\section{Current Technical Limitations and Future Directions \\ The most fundamental limitation in $4 \mathrm{D}$ thoracic CT} evaluation is that normal data and diagnostic criteria are not established yet. Another potential limitation is that radiation dose of $4 \mathrm{DCT}$ may be increased not only for a longer scan range to include the entire thorax, but also for the increased number of the evaluated respiratory phases. Consequently, the radiation dose of $4 \mathrm{D} C \mathrm{CT}$ needs to be further reduced by utilizing a new reconstruction algorithm such as a penalized weighted least square smoothing method allowing reduced image noise and preserved spatial resolution (27). Because image degradation may occur at high respiratory rates and great respiratory excursions, a higher temporal resolution of $4 \mathrm{DCT}$ is desirable under the circumstances. Post-processing software dedicated for free-breathing $4 \mathrm{D}$ thoracic $\mathrm{CT}$ is vital to enhance its clinical utility. Compared to conventional CT scanning, the markedly increased demand for image storage and workload, proportional to the tremendously increased number of $4 \mathrm{D}$ CT images, should be considered. Relatively short longitudinal scan coverage, e.g., $3.8 \mathrm{~cm}$ in a 64-detector scanner, may be limited in evaluating the whole thorax with $4 \mathrm{D} \mathrm{CT}$
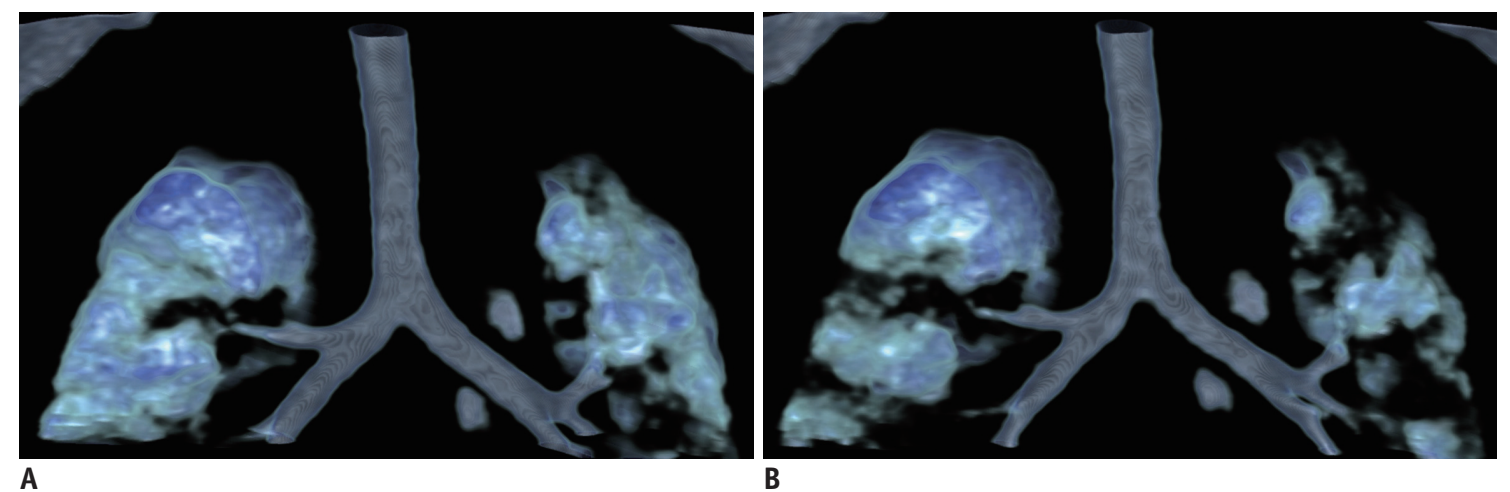

Fig. 9. Pulmonary air trapping in 14-day-old boy with repaired complete transposition of great arteries and vascular ring. Coronal inspiratory (A) and expiratory (B) volume-rendered CT images reveal multiple lung lesions with air trapping, suggesting small airway disease. Characteristic mosaic lung attenuation is shown on expiratory-phase $4 \mathrm{D}$ CT image (B). 
imaging, but it is feasible in small children. When $4 D C T$ scan is performed with intravenous injection of iodinated contrast agent, conscious sedation is usually required to maintain satisfactory image quality in young children. In contrast, sedation may be reduced or avoided in a noncontrast 4D CT scan.

\section{CONCLUSION}

Four-dimensional thoracic $\mathrm{CT}$ can provide useful functional information such as dynamic upper airway obstruction, tracheobronchomalacia, pulmonary air trapping, impaired diaphragm motion, and tumor invasion, in free-breathing children.

\section{Conflicts of Interest}

The author has no financial conflicts of interest.

\section{ORCID}

Hyun Woo Goo

https://orcid.org/0000-0001-6861-5958

\section{REFERENCES}

1. Lee EY, Mason KP, Zurakowski D, Waltz DA, Ralph A, Riaz F, et al. MDCT assessment of tracheomalacia in symptomatic infants with mediastinal aortic vascular anomalies: preliminary technical experience. Pediatr Radiol 2008;38:82-88

2. Long FR, Castile RG, Brody AS, Hogan MJ, Flucke RL, Filbrun $D A$, et al. Lungs in infants and young children: improved thin-section CT with a noninvasive controlled-ventilation technique--initial experience. Radiology 1999;212:588-593

3. Goo HW, Allmendinger T. Combined electrocardiography- and respiratory-triggered CT of the lung to reduce respiratory misregistration artifacts between imaging slabs in freebreathing children: initial experience. Korean J Radiol 2017;18:860-866

4. Goo HW. Combined prospectively electrocardiography- and respiratory-triggered sequential cardiac computed tomography in free-breathing children: success rate and image quality. Pediatr Radiol 2018;48:923-931

5. Lin L, Bivard A, Krishnamurthy V, Levi CR, Parsons MW. Wholebrain CT perfusion to quantify acute ischemic penumbra and core. Radiology 2016;279:876-887

6. Moorees J, Bezak E. Four dimensional CT imaging: a review of current technologies and modalities. Australas Phys Eng Sci Med 2012;35:9-23

7. Jahani N, Choi S, Choi J, Iyer K, Hoffman EA, Lin CL. Assessment of regional ventilation and deformation using 4DCT imaging for healthy human lungs during tidal breathing. $J$
Appl Physiol (1985) 2015;119:1064-1074

8. Wielpütz M0, Eberhardt R, Puderbach M, Weinheimer 0, Kauczor HU, Heussel CP. Simultaneous assessment of airway instability and respiratory dynamics with low-dose 4D-CT in chronic obstructive pulmonary disease: a technical note. Respiration 2014;87:294-300

9. Greenberg SB. Dynamic pulmonary CT of children. AJR Am J Roentgenol 2012;199:435-440

10. Goo HW. Current trends in cardiac CT in children. Acta Radiol 2013;54:1055-1062

11. Goo HW. Advanced functional thoracic imaging in children: from basic concepts to clinical applications. Pediatr Radiol 2013;43:262-268

12. Goo HW, Drubach L, Lee EY. Imaging techniques. In: Coley $\mathrm{BD}$, ed. Caffey's pediatric diagnostic imaging, 12th ed. Philadelphia, PA: Elsevier, 2013:506-518

13. Tan JZ, Crossett M, Ditchfield M. Dynamic volumetric computed tomographic assessment of the young paediatric airway: initial experience of rapid, non-invasive, fourdimensional technique. J Med Imaging Radiat Oncol 2013;57:141-148

14. Greenberg SB, Dyamenahalli U. Dynamic pulmonary computed tomography angiography: a new standard for evaluation of combined airway and vascular abnormalities in infants. Int $\mathrm{J}$ Cardiovasc Imaging 2014;30:407-414

15. Kwong Y, Mel A0, Wheeler G, Troupis JM. Four-dimensional computed tomography (4DCT): a review of the current status and applications. J Med Imaging Radiat Oncol 2015;59:545554

16. Goo HW. Four-dimensional CT of the diaphragm in children: initial experience. Korean J Radiol 2018;19:111-118

17. Goo HW. CT radiation dose optimization and estimation: an update for radiologists. Korean J Radiol 2012;13:1-11

18. Choong CK, Pasricha SS, Li X, Briggs P, Ramdave S, Crossett $M$, et al. Dynamic four-dimensional computed tomography for preoperative assessment of lung cancer invasion into adjacent structures. Eur J Cardiothorac Surg 2015;47:239-243; discussion 243

19. Goo HW. Individualized volume CT dose index determined by cross-sectional area and mean density of the body to achieve uniform image noise of contrast-enhanced pediatric chest CT obtained at variable kV levels and with combined tube current modulation. Pediatr Radiol 2011;41:839-847

20. Goo HW. Free-breathing cine CT for the diagnosis of tracheomalacia in young children. Pediatr Radiol 2013;43:922928

21. Goo HW, Kim HJ. Detection of air trapping on inspiratory and expiratory phase images obtained by 0.3 -second cine CT in the lungs of free-breathing young children. AJR Am J Roentgenol 2006;187:1019-1023

22. Kipritidis J, Hofman MS, Siva S, Callahan J, Le Roux PY, Woodruff $\mathrm{HC}$, et al. Estimating lung ventilation directly from 4D CT Hounsfield unit values. Med Phys 2016;43:33

23. Chavhan GB, Babyn PS, Cohen RA, Langer JC. Multimodality 
imaging of the pediatric diaphragm: anatomy and pathologic conditions. Radiographics 2010;30:1797-1817

24. Epelman M, Navarro OM, Daneman A, Miller SF. M-mode sonography of diaphragmatic motion: description of technique and experience in 278 pediatric patients. Pediatr Radiol 2005; 35:661-667

25. Akata S, Kajiwara N, Park J, Yoshimura M, Kakizaki D, Abe $K$, et al. Evaluation of chest wall invasion by lung cancer using respiratory dynamic MRI. J Med Imaging Radiat Oncol
2008;52:36-39

26. Hong YJ, Hur J, Lee HJ, Kim YJ, Hong SR, Suh YJ, et al. Respiratory dynamic magnetic resonance imaging for determining aortic invasion of thoracic neoplasms. J Thorac Cardiovasc Surg 2014;148:644-650

27. Li T, Schreibmann E, Thorndyke B, Tillman G, Boyer A, Koong A, et al. Radiation dose reduction in four-dimensional computed tomography. Med Phys 2005;32:3650-3660 